Internist 2009 $\cdot 50: 777-778$

DOI 10.1007/s00108-009-2428-5

Online publiziert: 04. Juni 2009

Springer Medizin Verlag 2009

\section{K. Werdan}

Universitätsklinik und Poliklinik für Innere Medizin III, Universitätsklinikum Halle (Saale) der Martin-Luther-Universität Halle-Wittenberg, Halle (Saale)
Liebe Leserinnen und Leser dieses Schwerpunktheftes „Sepsis“!

„Das Fieber, das verursacht wird durch eine Materie, die fault" (Hippokrates, 400 v. Chr.) stellt heutzutage das Hauptproblem unserer Intensivstationen dar: jeder 3. Intensivpatient leidet an einer Sepsis, und jeder 3. dieser Patienten wird an dieser Erkrankung versterben! Wie ist dies in einer Zeit möglich, in der die HerzKreislauf-Sterblichkeit innerhalb der letzten 20 Jahre halbiert worden ist? Warum können wir diese Erfolge nicht auch für die Sepsis vermelden?

\section{( Jeder 3. Intensivpatient leidet an einer Sepsis}

Ich möchte nicht zu viel versprechen: Auch nach dem Lesen der Beiträge dieses Schwerpunktheftes "Sepsis“ werden Sie natürlich nicht Antworten auf alle Fragen erhalten haben. Wenn Sie nach dem Lesen aber das Gefühl haben, dieses komplexe Krankheitsbild etwas besser zu verstehen, dann hätten wir Autoren das erreicht, was wir uns vorgenommen haben!

Vor knapp 100 Jahren - 1914 - hat der Internist Schottmüller in seinem Beitrag "Wesen und Behandlung der Sepsis" in den „Verhandlungen der Deutschen Gesellschaft für Innere Medizin" die auch heute noch gültige Definition der Sepsis gegeben: „Eine Sepsis liegt dann vor, wenn sich innerhalb des Körpers ein Herd gebildet hat, von dem konstant oder periodisch pathogene Bakterien in den Blutkreislauf gelangen, und zwar derart, dass durch diese Invasion subjektive und objektive Krankheitsbeschwerden ausgelöst werden." Schottmüller konnte allerdings noch nicht wissen, dass dem Patienten seine eigene „Abwehrreaktion“ gegen diese
Sepsis mindestens ebenso viele Probleme im Sinne einer überschießenden, aggressiven inflammatorischen „Autoimmunreaktion" machen kann wie die pathogenen Keime selbst. Diese Erkenntnis ist erst 1972 von L. Thomas mit „it is our response that makes the disease" konkretisiert worden.

Bakterientoxine und überschießende Inflammationsreaktion des Patienten kennzeichnen die komplexe Pathophysiologie der Sepsis, welche im Beitrag „Pathophysiologie und Keimspektrum der Sepsis" von Hauber u. Zabel anschaulich dargestellt wird. Aus meiner subjektiven Sicht sind die Entdeckung der „Tolllike-Rezeptoren" und die Aufdeckung der engen Vernetzung von Immunsystem und autonomem Nervensystem zwei der spannendsten Erkenntnisse der letzten Jahre auf diesem Gebiet: Die „Toll-like-Rezeptoren" vermitteln die Bindung von Toxinen und anderen Keimbestandteilen an die Körperzellen des Patienten und setzen damit die deletäre intrazelluläre Signaltransduktion mit Zellzerstörung in Gang. Ein intaktes autonomes Nervensystem kann als „cholinerger antiinflammatorischer Reflex" die überschießende Immunreaktion des Köpers in der Sepsis dämpfen. Leider besteht bei diesen Patienten eine hochgradige Einschränkung der Vagusaktivität, was diesen Schutzmechanismus anscheinend sehr stark schädigt.

Der Beitrag „Definition, Epidemiologie und ökonomische Aspekte der Sepsis bei Erwachsenen" von Moerer u. Quintel bringt uns eindrücklich ins Bewusstsein, wie gravierend das Problem der Sepsis tatsächlich ist. Bemerkenswert ist die Aussage der Autoren, dass wohl jeder 2. Bundesbürger den Begriff "Sepsis“ noch nie gehört hat - über den „Herzinfarkt" wissen dagegen sicherlich die meisten Ihrer Patienten Bescheid, obwohl die Zahl der Erkrankungen und Todesfälle mit 150.00o Sepsiserkrankungen und 60.00o Sepsistodesfällen jährlich in Deutschland bei beiden Erkrankungen vergleichbar ist. Und es kommt noch schlimmer: Die Sepsissterblichkeit hat sich in den letzten 20 Jahren verdoppelt, eine Folge der demographischen Entwicklung. Grund genug, den wichtigen Informationen des Beitrags von Moerer u. Quintel Beachtung zu schenken.

Jeder 2. derjenigen Patienten, welche an der Sepsis versterben, verstirbt an den Folgen des septischen Schocks. Dieser „septische Schock" wird üblicherweise als „septischer Kreislaufschock“ angesehen. In unserem Beitrag „Septischer Kreislaufschock und septische Kardiomypathie" wollen wir aufzeigen, dass nicht nur der Kreislaufschock, sondern auch die Schädigung des Herzens in der Sepsis - die viel zu selten diagnostizierte ,septische Kardiomyopathie" - entscheidend zur ungünstigen Prognose der Sepsis beiträgt.

Mit der Etablierung von Sepsisleitlinien in den letzten 5 Jahren hat die Betreuung der Sepsispatienten ein sehr viel solideres und evidenzbasierteres Fundament erhalten, als dies bisher der Fall war. In Deutschland hat die Leitlinie „Diagnose und Therapie der Sepsis" der Deutschen Sepsis-Gesellschaft und der Deutschen Interdisziplinären Vereinigung für Intensiv- und Notfallmedizin (DIVI) seit ihrer Publikation 2006 erfreulicherweise bereits eine sehr große Verbreitung gefunden. Man kann schon gespannt auf die Leitlinienaktualisierung sein, welche in den nächsten Monaten publiziert werden wird. Insofern bin ich den Initiatoren 
dieser Leitlinie, den Kollegen Brunkhorst u. Reinhart, ganz besonders dankbar, dass sie die beiden Beiträge „Diagnose und kausale Therapie der Sepsis“ und „Supportive und adjunktive Therapie der Sepsis" für dieses Themenheft übernommen haben.

\section{() Wir haben den Fokus bisher viel zu wenig auf die Frühstadien der Sepsis gerichtet}

Wenn wir in Deutschland vom „Sepsispatienten" sprechen, dann meinen wir in der Regel damit den Intensivpatienten mit schwerer Sepsis (Sepsis mit Organversagen) oder septischem Schock und nicht wie eigentlich entsprechend der internationalen Sepsisklassifizierung vorgegeben den Patienten mit „einfacher“ Sepsis, d. h. mit „Sepsis ohne Organversagen“. Mit den Intensivpatienten mit schwerer Sepsis oder septischem Schock müssen wir bedauerlicherweise eine sehr hohe Letalität und sehr viele enttäuschende Therapiestudien assoziieren, was einem in Anbetracht des bei diesen schwersten Sepsisstadien schon „aus dem Ruder“ gelaufenen Immunsystem eigentlich nicht verwundert.

Aus meiner Sicht haben wir den Fokus bisher viel zu wenig auf die Frühstadien der Sepsis gerichtet, auf diejenigen Patienten mit ambulant erworbener Sepsis, welche in der Notaufnahme ärztliche Hilfe suchen. Häufig werden diese Patienten mit bereits nachweisbarer Sepsis beispielsweise noch als „Pneumoniepatienten“ und nicht als „Patienten mit pneumogener Sepsis" betrachtet und demzufolge auch nicht so rasch als möglich entsprechend den Sepsisleitlinien behandelt. Mit dieser Sepsisgruppe und den Möglichkeiten einer Optimierung der Behandlung beschäftigt sich schwerpunktmäßig der letzte Beitrag „Spezielle Aspekte bei Sepsispatienten: Initiale Phase auf der Notaufnahme, Lebensalter, Geschlecht, Postintensivphase" von Müller-Werdan et al.
Liebe Leserinnen und Leser,

die Sepsis hat viele Gesichter. Autoren und Herausgeber haben versucht, einige Schnappschüsse in diesem Themenheft für Sie abzubilden. Wir hoffen, wir konnten Ihnen einige aktuelle und praxisrelevante Aspekte dieses Krankheitsbilds vermitteln.

Eine informative Lektüre wünscht Ihnen

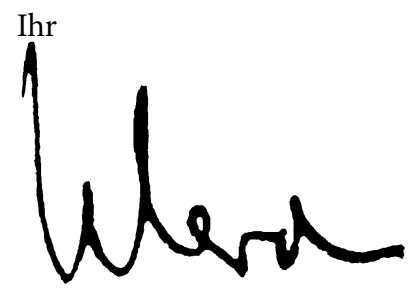

K. Werdan

\section{Korrespondenzadresse}

\section{Prof. Dr. K. Werdan}

Universitätsklinik und Poliklinik für Innere Medizin III, Universitätsklinikum Halle (Saale) der Martin-Luther-Universität Halle-Wittenberg Ernst-Grube-Straße 40, 06120 Halle (Saale)

karl.werdan@medizin-uni-halle.de

Interessenkonflikt. Der korrespondierende Autor gibt an, dass kein Interessenkonflikt besteht.

\section{Bakterien verdichten Gallenstents}

Eine unangenehme Folgeerscheinung verschiedener Krebsarten im Bauchraum sind blockierte Gallengänge. Abhilfe leisten Stents. In den Kathetern siedeln sich jedoch Bakterien an und bilden Biofilme. In innen sind die Mikroorganismen vor einer Abwehr durch das Immunsystem oder gegen Antibiotika geschützt.

Forscher des Helmholtz-Zentrums für Infektionsforschung (HZI) aus Braunschweig haben nun die Gallenstents von zahlreichen Patienten untersucht. Obwohl sich die Bakteriengemeinschaften unterscheiden, konnten die Forscher Hauptbesiedler, wie das Bakterium Streptococcus anginosus, identifizieren. Wechselwirkungen und Abhängigkeiten unter den Bakterien erfassten die Forscher außerdem statistisch. Dabei stellte sich heraus, dass die Besiedlung der Stents Prinzipien folgt, die denen der Biofilm-Bildung von zum Beispiel Zahnbelag ähneln. Dieses Wissen soll in Zukunft die Entwicklung von Medikamenten erleichtern, die zielgerichtet gegen die Biofilme wirken.

Literatur: Scheithauer BK, Wos-Oxley ML, Ferslev B et al (2009) Characterization of the complex bacterial communities colonizing biliary stents reveals a hostdependent diversity. ISME J Epub ahead of print

Quelle:

Helmholtz-Zentrums für Infektionsforschung (HZI), www.helmholtz-hzi.de 